\title{
Caracterización de la agricultura familiar campesina, comuna de Cayes-Jacmel, Haití
}

\author{
Characterization of peasant family farming, Cayes-Jacmel Commune, Haiti \\ Rolord Severe ${ }^{1}$, María Beatriz Vera $O .^{2^{*}}$
}

\begin{abstract}
RESUMEN
La pequeña agricultura es un tema que ha llamado la atención de muchas entidades estatales, locales, regionales e internacionales durante los últimos años, por su importancia en la producción de alimentos y la reducción de la pobreza en las comunidades rurales del mundo. En este contexto la presente investigación tuvo como objetivo caracterizar socioeconómica y productivamente a la pequeña agricultura en la sección comunal de Cap-Rouge (Cayes-Jacmel), Haití. La metodología empleada correspondió a un estudio de caso, recolectando información mediante una encuesta estática y semiabierta, aplicada a 36 pequeños agricultores de ambas zonas agroecológicas (cálida y moderada) y una entrevista, aplicada a los expertos claves (los líderes de organización comunitaria, los representantes del centro de formación en agroecología, los profesionales de ONG y representantes del Estado). Los resultados permiten destacar una edad media de 49 años para los pequeños agricultores en la sección, con una baja participación de mujeres como agricultoras. Más de la mitad de los agricultores se incorporan a esta actividad por tradición familiar. En relación con los aspectos económicos, la actividad que más aporta es la pecuaria, donde gallinas y cerdos aportan el 37,9\% y $33,47 \%$, respectivamente. El $27,78 \%$ de los pequeños agricultores supera los 50.000 gourdes al año (equivalente a $\$ 1.200$ US). Respeto de los aspectos productivos, se indica que el 80,56\% de los campesinos son propietarios de la tierra, cuyo promedio es inferior a una hectárea.
\end{abstract}

Palabras clave: caracterización socioeconómica y productiva, pequeña agricultura

\begin{abstract}
Small-scale agriculture is a topic that has drawn the attention of many State agencies, local, regional and international during the last few years, due to their importance in the food production and poverty reduction in rural communities of the world. In this context, the present investigation had as its objective to characterize socio-economically and productively to the small-scale agriculture in the communal section of Cap Rouge (Cayes-Jacmel). The methodology used, it connected with a study case, collecting information through a static and semi-open survey applied to 36 small farmers and an interview applied to the key experts (the leaders of community-based organization, the representatives of the agroecology training center, the NGOs'professionals and representatives of the State). The results allow highlighting an average age of 49 years for small farmers in the section, with a low participation of women as farmers. More than half of them are incorporated by family tradition. In relation to the economic aspects, the activity in more carrier item is the livestock with hens and pigs of $37.9 \%$ and $33.47 \%$ respectively and the 27.78 per cent of small farmers exceeds the 50,000 gourdes per year (equivalent to $\$ 1200$ US). In addition, the productive aspects indicate that $80.56 \%$ of the farmers are landowners whose average is less than one hectare.
\end{abstract}

Key words: characterization socioeconomic and productive, small-scale agriculture

\section{Introducción}

La producción de alimentos en los últimos años a nivel mundial viene siendo una preocupación para la gran mayoría de los gobiernos y las organizaciones que se ocupan de la alimentación. A pesar de los grandes logros en la productividad, son 925 millones de personas que, según se estima, pasaron a engrosar las filas de quienes padecen hambre en el mundo, y $70 \%$ de ellas reside en las zonas rurales (Organización para la Agricultura y la Alimentación (FAO), 2009). En estas circunstancias la agricultura, en particular las pequeñas explotaciones agrícolas, tendrá que desempeñar un papel mucho más eficaz en esos países, por lo que representa en la actualidad un factor clave del sustento en la mayoría de las

1 Facultad de Ciencias Agrarias, Escuela de Graduados, Universidad Austral de Chile, Independencia 641, Valdivia, Chile.

2 Instituto de Economía Agraria, Facultad de Ciencias Agrarias, Universidad Austral de Chile, Independencia 641, Valdivia, Chile, fono: 56-63-2221235.

* Autor para correspondencia: bvera@uach.cl

Fecha de Recepción: 8 Mayo, 2014.

Fecha de Aceptación: 8 Junio, 2014. 
comunidades más pobres del mundo (Instituto Interamericano de Cooperación para la Agricultura (IICA), et al., 2009).

En América Latina hacia 1990 había aproximadamente 17 millones trescientas mil unidades agropecuarias, donde $85,8 \%$ de ellas podían considerarse como unidades campesinas y el $14,2 \%$ como unidades empresariales. De las unidades campesinas $62 \%$ eran unidades con recursos insuficientes y $23,8 \%$ pequeñas propiedades agropecuarias con suficientes recursos. Para Centroamérica los estudios realizados por Baumeister (2001) indican que del 69\% de explotaciones campesinas y pequeñas, $25 \%$ de las fincas con $14 \%$ de la superficie podía considerarse pequeña producción intensiva.

En Haití, desde hace pocos años la agricultura se encuentra inmersa en un proceso de cambio profundo e inevitable, y las principales razones que han impulsado este cambio han sido de índole social, política y económica, lo que influye sobre el acceso y la disponibilidad de recursos financieros y tecnológicos para continuar desarrollándose. Un estudio reciente en Haití revela que la superficie agrícola útil es de 1.358 .508 ha, en el departamento del Artibonite, siendo la más importante con $12 \%$ del total, mientras que la más pequeña se encuentra en la parte sureste con $1 \%$ del total (Ministerio de la Agricultura de Recursos Naturales y del Desarrollo Rural (MARNDR), 2012). Según el Instituto de Estadística Haitiana de Informática (IHSI), (2007), casi la mayoría de las familias rurales son micropropietarias, y el $50 \%$ de las 800.000 explotaciones agrícolas existentes no alcanzan a superar las 1,29 hectáreas (Victor, 2010).

De acuerdo con lo anterior, esta investigación se planteó el objetivo de caracterizar socioeconómica y productivamente a la pequeña agricultura en la sección comunal de Cap-Rouge (Cayes-Jacmel), Haití.

\section{Materiales y Métodos}

\section{Área de estudio}

El estudio se llevó a cabo en Cap-Rouge, una sección rural de la comuna "Cayes Jacmel" situada en el departamento sureste de Haití. Presenta una superficie de $15 \mathrm{~km}^{2}$ repartida en 23 asentamientos poblacionales donde alojan 18.000 habitantes agrupados en 2.500 familias, el $85 \%$ (2.125) son pequeños agricultores (IHSI, 2009; Jean-Baptiste, 2012; MARNDR, 2012).

Universo y muestra de estudio. El universo de estudio está constituido por todos los agricultores de la sección de Cap-Rouge:

$\checkmark$ Que tenga un área menor de 1 carreau destinada a la agricultura (unidad de medida de superficie de la tierra muy usada en Haití y que equivale a $12.900 \mathrm{~m}^{2}$ o $1,29 \mathrm{ha}$ ). (IHSI, 2007).

$\checkmark$ Que se comercialice por lo menos una parte de la producción.

Debido a que existe una marcada diferencia agroecológica, en el Tabla 1 se indica la distribución de la muestra en ambas zonas.

\section{Metodología}

La metodología para la obtención de la información fue el sondeo tipo "Snowball", este consiste en localizar a algunos individuos, estos conducen a otro, y estos a otros y así hasta conseguir datos relevantes, utilizando como punto de partida los mismos grupos de pequeños agricultores pertenecientes a las redes de organizaciones campesinas (Torres, 2005). Los instrumentos utilizados para la obtención de información sobre la pequeña agricultura en la sección fueron: una encuesta estática, semiabierta aplicada a los 36 pequeños agricultores de 12 asentamientos y un cuestionario a 5 expertos de la zona cálida y 7 de la zona moderada, que conformaron la muestra. Los datos fueron analizados a base de estadística descriptiva (Cascos, 2004).

\section{Resultados y Discusión}

\section{Caracterización sociodemográfica de los agricultores}

a) Edad. Se encontró un promedio de edad de 49 años para los pequeños agricultores en la sección comunal de Cap-Rouge, donde 22,22\% y $55,55 \%$ de los encuestados en la zona moderada y cálida, respectivamente, tiene entre 36 y 50 años (Tabla 2). Se observó en la zona moderada donde hay $11,11 \%$ que son mayores de 65 años. Sanon (2010) encontró en esta zona que la mayoría de los agricultores son mayores de edad, señalando que la migración de los jóvenes y mujeres a la ciudad y a la república vecina son las causales. 
Tabla 1. Distribución de la muestra en la zona de Cap-Rouge, Haití.

\begin{tabular}{llcc}
\hline \multirow{2}{*}{ Zona agroecológica } & \multicolumn{1}{c}{ Asentamientos } & $\begin{array}{c}\text { Agricultores en la } \\
\text { muestra (n) }\end{array}$ & $\begin{array}{c}\text { Agricultores } \\
\text { a encuestar (n) }\end{array}$ \\
\hline Cálida & Canyette, Pierre, Ogé, Desmarates, Savarie, La source & 35 & 18 \\
Moderada & Grande terre, Neptune, Vergeon, Clemence I, Jeanty, & 35 & 18 \\
Total & Clemence II & 70 & 36 \\
\hline
\end{tabular}

Tabla 2. Distribución de los pequeños agricultores de Cap-Rouge, según edad.

\begin{tabular}{cccccc}
\hline \multirow{2}{*}{ Edad (años) } & \multicolumn{2}{c}{ Zona moderada } & & \multicolumn{2}{c}{ Zona cálida } \\
\cline { 2 - 3 } \cline { 5 - 6 } & No & $\%$ & 11,11 & & No \\
\hline $18-35$ & 2 & 22,22 & & 3 & 16,67 \\
$36-50$ & 10 & 55,56 & & 10 & 55,55 \\
$51-65$ & 2 & 11,11 & & 5 & 27,78 \\
$>65$ & 18 & 100 & & 0 & 100 \\
Total & 18 & & & 18 & 0 \\
\hline
\end{tabular}

b) Participación por sexo. A pesar de una proporción dominante de mujeres en la sección de Cap-Rouge (52\%) (DSDS y IHSI, 2012), en la Figura 1 se observa solo una proporción de $22,22 \%$ de mujeres en la zona moderada que se dedican a la actividad de la agricultura, mientras que la participación de los hombres alcanza una proporción de $77,78 \%$. El resultado no difiere en la zona cálida donde el $44,44 \%$ son mujeres y el $55,56 \%$ son hombres que se dedican a la pequeña agricultura.

La mayoría de las mujeres se dedican más a las labores domésticas en casa o a las actividades de comercio (Gédéon, 2010; Sanon, 2010).

c) Escolaridad. En la Tabla 3 se observa una proporción de 33,33\% de los encuestados de la zona moderada que no han tenido acceso a una formación escolar, $27,78 \%$ de ellos no ha terminado su primaria, igual proporción $(27,78 \%)$ completó su primaria y $11,11 \%$ tiene un nivel secundario incompleto. No hay pequeños agricultores con niveles de escolaridad secundaria completa, ni superior.

Por otro lado, en la zona cálida existe una proporción de 55,56\% de pequeños agricultores que no cuenta con una formación escolar, $27,78 \%$ con nivel de escolaridad primaria incompleta, $11,11 \%$ termina su etapa primaria y $5,56 \%$ con un nivel secundario incompleto. Como se puede observar, al igual de la zona moderada, los agricultores de la zona cálida no finalizan su secundaria y no cuentan con un nivel superior de educación.
Es importante señalar ciertas similitudes que presenta el resultado encontrado por Fanfan (2012) en un estudio realizado con el movimiento campesino de Papaye de la provincia central del país, donde obtiene una alta proporción (40\%) de campesinos sin educación formal, es decir, son analfabetos, $35 \%$ de ellos termina su primaria, $20 \%$ con un nivel primario incompleto.

d) Razones de incorporación y tiempo dedicado a la pequeña agricultura. La tradición familiar $(63,89 \%)$ es una de las principales razones que han motivado a los agricultores de Cap-Rouge a dedicarse a la actividad de la agricultura, lo que concuerda con Gédéon (2010), quien señala que la mayoría de los agricultores en la zona de Cap-Rouge se incorporan en la agricultura gracias a la tradición de sus padres que lo llevan al campo.

También existe una proporción de $25 \%$ de los encuestados que realizan esta actividad por el motivo de abastecer su hogar con alimentos, mientras que $11,11 \%$ de ellos se incorpora a la actividad para sostener económicamente sus hogares. De los agricultores encuestados no se reveló ninguna otra razón que lo motive a incorporarse a la agricultura (Figura 2).

Respeto de la hora dedicada para realizar la actividad del predio se identifica 5,56\% de agricultores, en ambas zonas, que trabajan entre 1 a 2 días a la semana. Asimismo, se observa que el $38,89 \%$ de los encuestados en la zona cálida y el $33,33 \%$ de la zona moderada dedican entre 3 a 4 


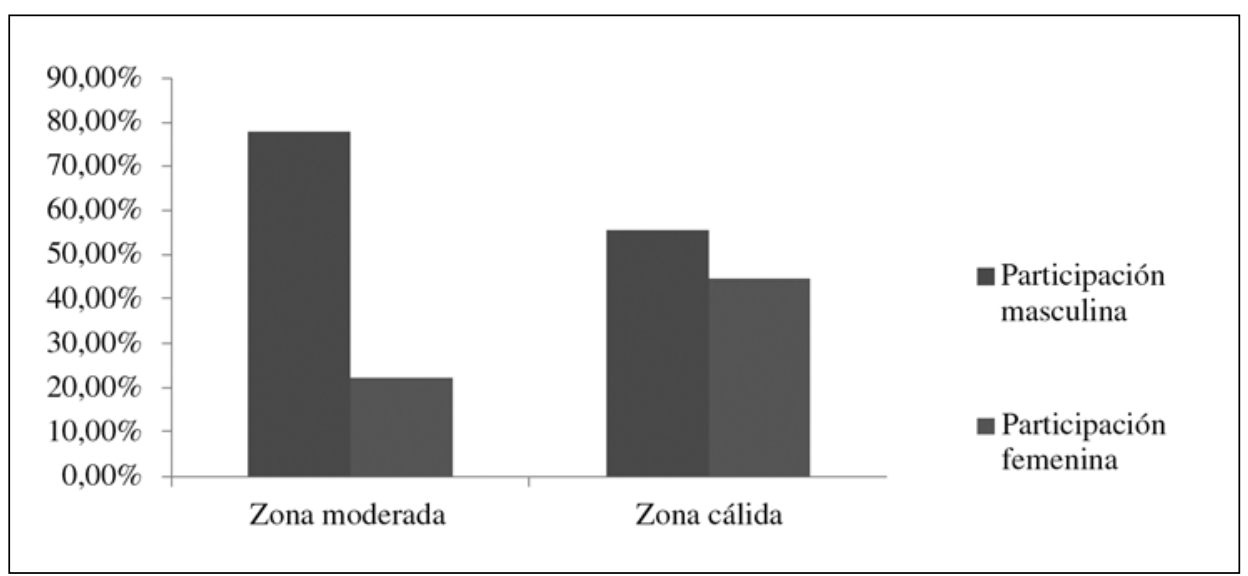

Figura 1. Distribución de los pequeños agricultores por zona, según sexo.

Tabla 3. Nivel de escolaridad de los pequeños agricultores en Cap-Rouge.

\begin{tabular}{|c|c|c|c|c|}
\hline \multirow{2}{*}{ Escolaridad } & \multicolumn{2}{|c|}{ Zona moderada } & \multicolumn{2}{|c|}{ Zona cálida } \\
\hline & $\mathrm{N}$ & $\%$ & $\mathrm{~N}$ & $\%$ \\
\hline Sin escolaridad & 6 & 33,33 & 10 & 55,56 \\
\hline Primaria incompleta & 5 & 27,78 & 5 & 27,78 \\
\hline Primaria completa & 5 & 27,78 & 2 & 11,11 \\
\hline Secundaria incompleta & 2 & 11,11 & 1 & 5,56 \\
\hline Secundaria completa & 0 & 0,00 & 0 & 0,00 \\
\hline Técnico & 0 & 0,00 & 0 & 0,00 \\
\hline Universitario & 0 & 0,00 & 0 & 0,00 \\
\hline Total & 18 & 100 & 18 & 100 \\
\hline
\end{tabular}

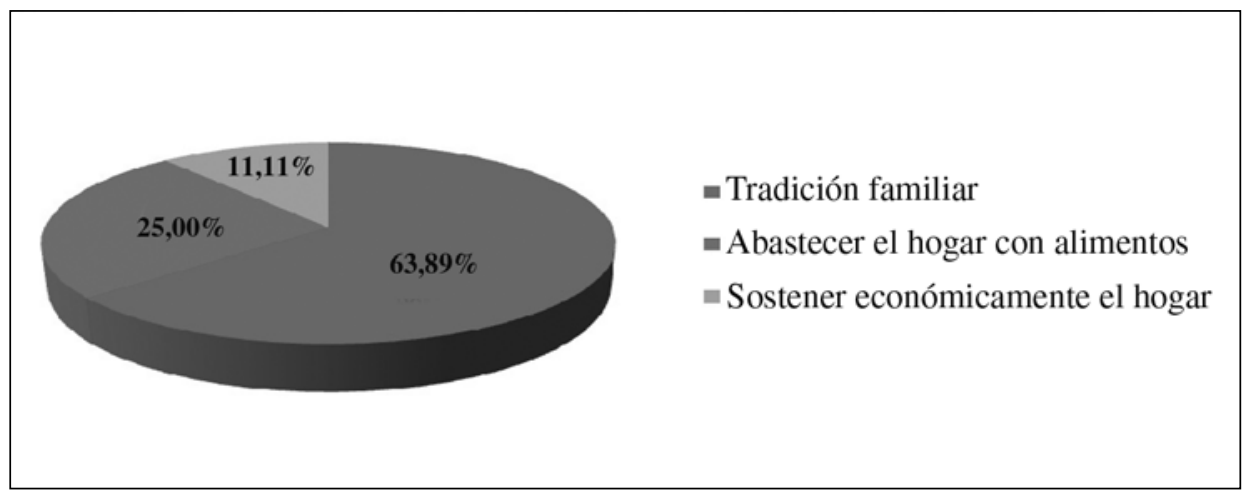

Figura 2. Motivos de inserción en la pequeña agricultura.

días semanalmente al trabajo de campo. La mayor proporción de los pequeños agricultores encuestados tanto en la zona cálida $(55,56 \%)$ como en el caso de la zona moderada $(66,11 \%)$ trabajan en sus predios entre 5 y 6 días a la semana. Es importante señalar que no existen agricultores que trabajen los 7 días de la semana, debido a que muchos de ellos descansan los domingos, van a la iglesia y/o se reúnen en familia (Figura 3 ).

e) Participación en la organización comunitaria. La asociación entre las familias pobres es fundamental para mejorar sus medios de vida, fortalecer la unidad, la confianza y cohesión social y desarrollar iniciativas más complejas, con 


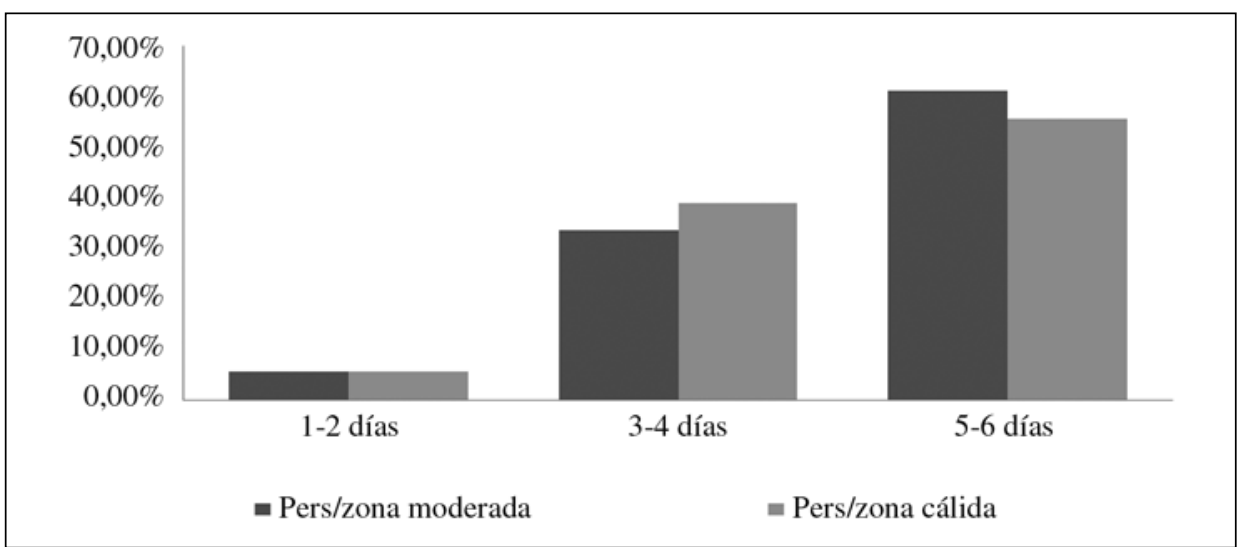

Figura 3. Tiempo dedicado a las labores de campo.

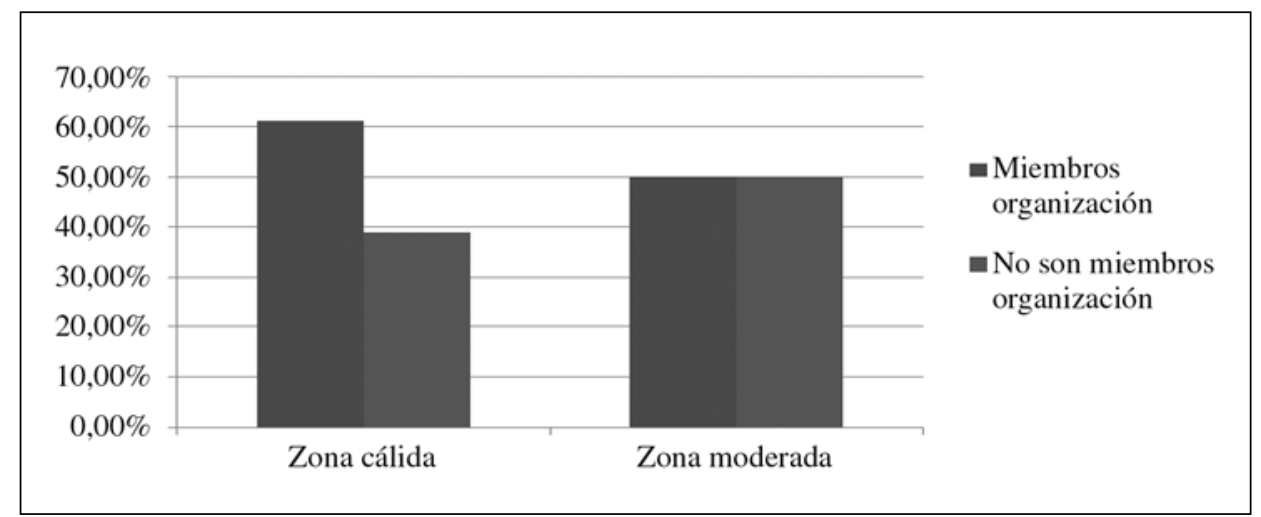

Figura 4. Participación en organizaciones comunitarias.

resultados que le dan sentido de pertenencia social y económica (FAO,2012).

En la zona cálida de Cap-Rouge hay $61,11 \%$ de agricultores encuestados que pertenecen a una organización comunitaria de base y $38,89 \%$ de estos afirman que no pertenecen a ningún grupo comunitario en la zona. Por lo tanto, en la zona moderada se observa que 50\% de los agricultores participa en una organización y el otro $50 \%$ no participa (Figura 4).

\section{Caracterización económica de los agricultores}

a) Actividad agrícola. En la sección comunal de Cap-Rouge existe una gama de variedades de cultivos que explotan los agricultores en las dos zonas agroecológicas y que permiten observar ciertas diferencias entre una zona respeto de la otra. Como se puede observar en la Figura 5, hay 25\% de explotaciones en la zona moderada, y $12 \%$ en la zona cálida que cultivan hortalizas. Respeto de la zona moderada, la condición climática se ve muy favorable a la explotación de estas especies, por la baja temperatura.

En cuanto a la producción de granos, la situación es contraria a lo precedente, ya que estos ocupan una proporción de $15 \%$ dentro de la gama de cultivos explotados en la zona moderada, frente a $34 \%$ en la zona cálida, siendo los principales granos que cultivan los pequeños agricultores en esta sección, el maíz y el sorgo. Estos dos cultivos han sido muy explotados en la zona cálida, debido a la condición climática favorable junto con más incidencia de la luz sobre la planta, por lo que hay menos bosques que dan sombra. Se puede observar también 15\% y $16 \%$ de producción de leguminosas en las zonas moderada y cálida, respectivamente. En lo que respecta a la producción de raíces y tubérculos en la zona moderada hay $18 \%$, mientras que en la zona cálida 24\% (Figura 5). 


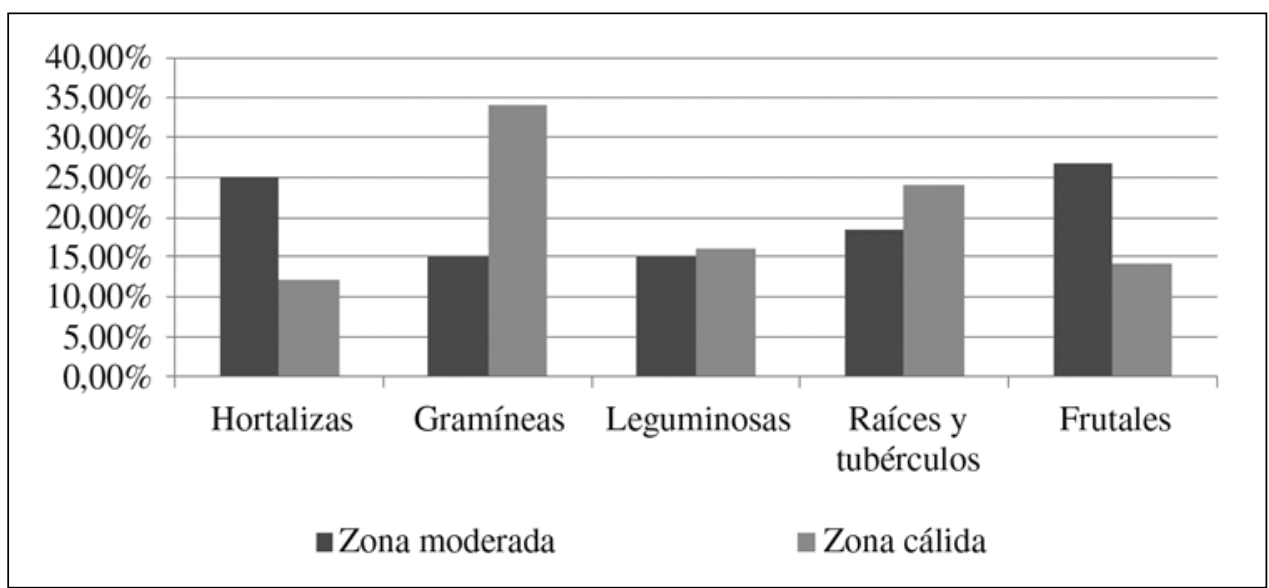

Figura 5. Actividad agrícola en el predio.

Además, se considera como especie importante los frutales, que ocupan una proporción de $26,67 \%$ de las explotaciones en la zona moderada y una porporción de $14 \%$ para la zona cálida. Esta baja presencia de frutales en la zona cálida es causa de la sobreexplotación de los bosques en esta parte, para la fabricación de carbón y de madera. Según Pierre (2010), el carbón vegetal representa hasta 30\% en los ingresos anuales de la economía familiar campesina.

b) Actividad pecuaria. Los resultados encontrados permiten afirmar que los pequeños agricultores crían $27,42 \%$ de ganado menor, como cordero y cabrito; $0,81 \%$ de vacuno, y una proporción de $0,40 \%$ de otros tipos de animales, como caballo, mula y burro, que los pequeños agricultores crían para el transporte de cosecha y materiales pesados. Es importante señalar que a pesar del gran efecto causado por ciertas enfermedades en la crianza de los animales dentro de la sección, se destaca la actividad pecuaria con gallinas y cerdos de $37,90 \%$ y $33,47 \%$, respectivamente.

En cuanto a la venta de animales, $27,78 \%$ de los agricultores encuestados en la zona moderada tiene un nivel de venta entre 0 y 3 animales al año, $44,44 \%$ con un nivel de venta entre 4 y 6 animales, $11,11 \%$ entre 7 y 10 animales y $16,67 \%$ venden 11 o más animales al año.

Por su parte, en la zona cálida se encontró una proporción de $22,22 \%$ de pequeños agricultores con nivel de venta entre 0 y 3 animales al año, 38,89\% venden entre 4 y 6 animales, 33,33\% entre 7 y 10 animales y una proporción de 5,56\% agricultores con un nivel de venta de 11 o más animales al año.
En ambas zonas agroecológicas se constata que la mayor parte de los agricultores tienen su tramo de venta entre 4 y 6 animales.

Es importante señalar que la gran mayoría de los pequeños agricultores realizan la venta de sus productos en el mercado local y en muchos casos por medio de un intermediario.

c) Aporte de la actividad agrícola y pecuaria en el hogar. El 19,44\% de los agricultores encuestados en la zona de Cap-Rouge tiene un nivel de venta menor de $25 \%, 25 \%$ de ellos afirman que su nivel de venta se mantiene entre $26 \%$ y $50 \%$.

La mitad de los encuestados obtienen un nivel de venta entre $51 \%$ y $75 \%$, mientras que solo $5,6 \%$ accede a un nivel de venta superior al $75 \%$ (Figura 6).

Se puede observar que $5,56 \%$ de los agricultores encuestados con ingresos entre 0 a 1.000 gourdes (según la tasa de cambio en el Banco de la República de Haití 1 dólar es igual a 42,5 gourdes), mientras que ninguno de ellos ha obtenido ventas que van desde 1.001 a 10.000 gourdes.

También entre 10.001 a 20.000 gourdes se ubica el $5,56 \%$ de los encuestados; entre 20.001 y 30.000 está el $11,11 \%$ de los agricultores encuestados; entre 30.001 y 40.000 están los ingresos de 22,22\% de los encuestados; entre 40.001 y 50.000 gourdes se sitúa el ingreso de $19,44 \%$ de los encuestados, en un ingreso mayor de los 50.000 gourdes está la mayoría $(27,78 \%$ ) de ellos, mientras que $8,33 \%$ de los agricultores encuestados en la zona de Cap-Rouge ignora el rango de ingreso obtenido a partir de la venta de productos del predio (Figura 7). 


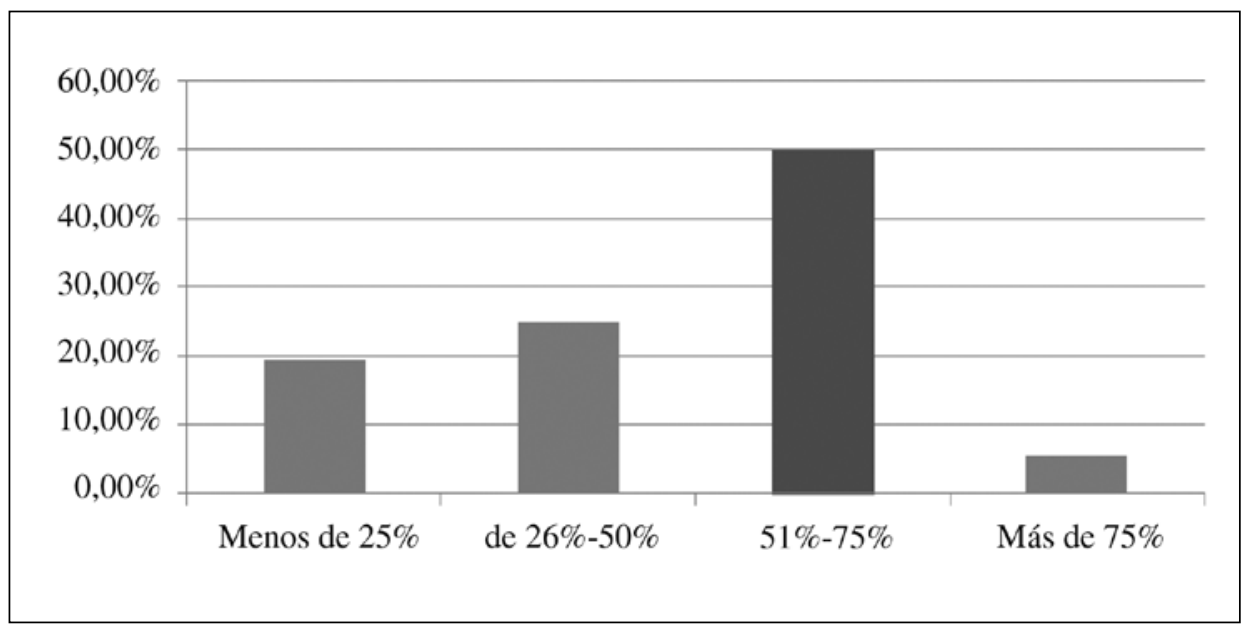

Figura 6. Nivel de venta de los agricultores.

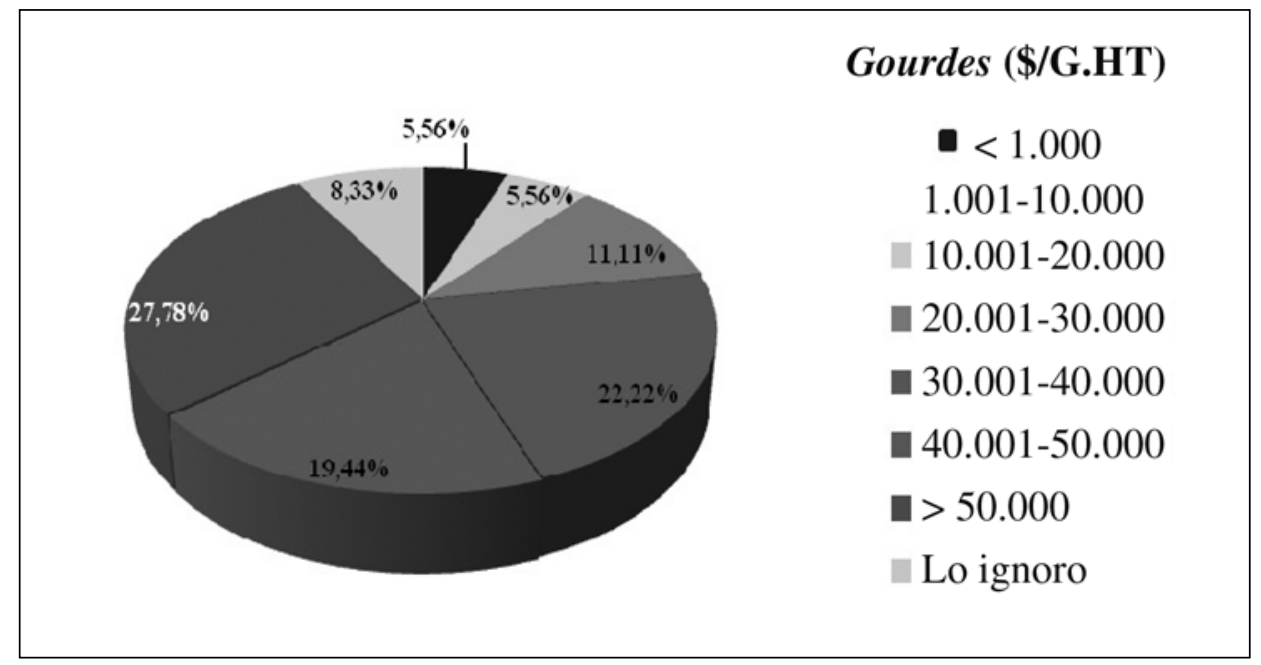

Figura 7. Ingresos económicos de los agricultores.

\section{Caracterización productiva de los agricultores}

a) Tenencia y origen de la tierra. La propiedad del terreno es lo que más conflicto genera en la sociedad haitiana (Victor, 2010), sin embargo, esta situación no ha impedido que muchos agricultores tengan la propiedad de sus terrenos.

Se observó que el 80,6\% de los agricultores encuestados declararon ser propietarios de su terreno, mientras que $13,9 \%$ trabajan espacios por aparcería y el 5,6\% de ellos arrienda superficies para instalar sus actividades.

En un estudio realizado por Inter Entreprise S.A. (INESA) (2001), citado por Jean Baptiste (2007), el $77 \%$ de los agricultores son propietarios de sus parcelas, contra $5,4 \%$ que trabaja por aparcería y el resto explota tierras pertenecientes al Estado.

Se identifica que el $44,44 \%$ de los agricultores encuestados obtiene sus tierras por herencia familiar y $16,67 \%$ por compra.

Asimismo, existe $36,11 \%$ que a pesar de ser herederos de tierras por parte de sus familias han realizado ciertas compras de tierra para explotar. Se indica también $2,78 \%$ de los encuestados que por muchas razones económicas y sociales explotan parcelas de tierra sin ningún requisito legal (Figura 8).

b) Superficie utilizada y recursos disponibles. Los criterios de distinción de pequeño agricultor se funda en la cantidad de hectáreas efectivamente utilizadas, de acuerdo con la normativa del IHSI 


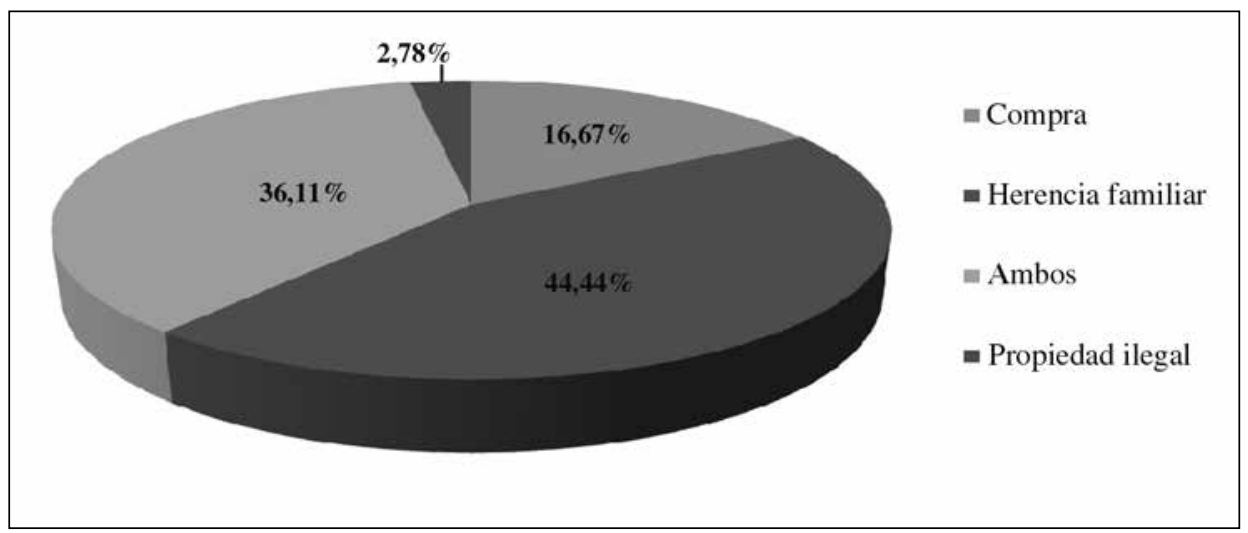

Figura 8. Origen de la adquisición de la tierra.

(2003). Aquellos que poseen una superficie igual o bajo las 1,29 hectáreas es considerado como grupo objetivo de este informe. En esta investigación se obtuvo una superficie promedio de 0,83 ha para los agricultores de la zona moderada y 0,68 ha para los de la zona cálida. Este resultado concuerda con Dure (2008), quien encuentra en una zona rural características similares a la de Cap-rouge, $48 \%$ de la población de pequeños agricultores que explotan predios de 0,65 ha de superficie, de malas características y que solo sirve para asegurar su medio de subsistencia.

En cuanto a los recursos, los agricultores cuentan solo con las herramientas manuales para la realización de las labores de campo, sin acceso a maquinaria, y un punto para almacenar los stocks, etcétera.

Además, para explotar su predio el 16,67\% de los agricultores utiliza su propio capital para financiar la actividad del predio, el 25\% de ellos obtiene dineros provenientes de familiares que viven en el exterior, $2,78 \%$ usan créditos del sector privado (bancos, cooperativas, etc.). Una proporción idéntica a la anterior obtiene fondos del organismo estatal, mientras que $13,89 \%$ de los encuestados se beneficia de fondos por parte de las ONG nacionales como internacionales, para explotar sus predios. Para iniciar la actividad del predio $44,44 \%$ de los encuestados recurre a otras vías tales como donación, préstamo informal entre amigos de la comunidad, recolección de dinero por parte de clientes para devolver en productos agrícolas, etc. (Figura 9).

c) Actividad con mayor aporte económico para el predio. El 38,89\% de los agricultores que tiene actividad pecuaria es la que más contribuye a la economía en la sección rural de Cap-Rouge, lo que refuerza la afirmación de los expertos del

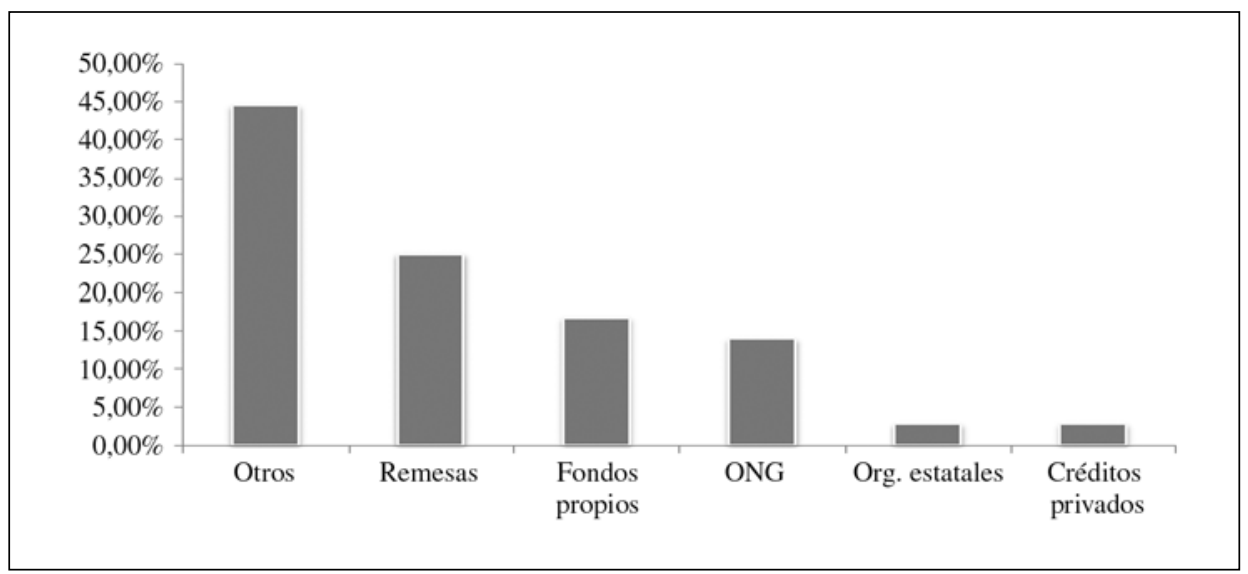

Figura 9. Fuentes de financiamiento para las actividades del predio. 


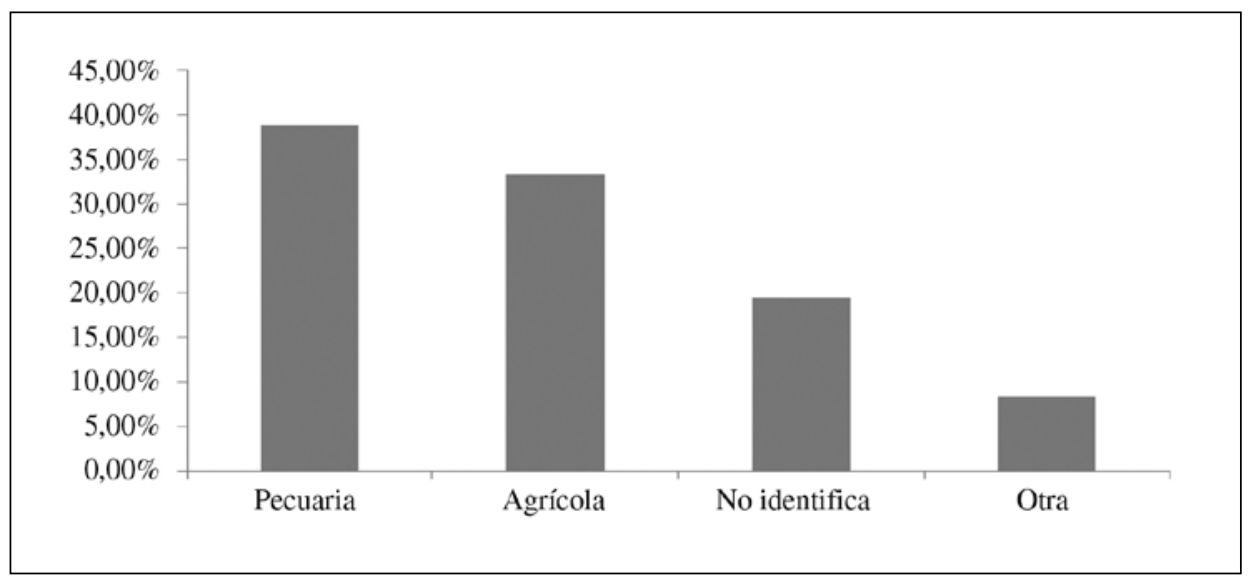

Figura 10. Actividad que aporta a la economía familiar.

Ministerio de la Agricultura, quienes sostienen que la actividad pecuaria es el ahorro de los campesinos rurales (MARNDR, 2010).

Por otro lado, el 33,33\% de los encuestados considera la actividad agrícola como el rubro portador de los ingresos en su hogar y el 19,44\% de ellos no tiene la menor idea sobre el principal rubro portador de ingresos. Asimismo, el 8,33\% de estos agricultores afirma que el mayor ingreso proviene de otro sector (Figura 10).

\section{Conclusiones}

La información analizada en este estudio permite concluir que la edad promedio de los pequeños agricultores en la sección comunal de Cap-Rouge, Haití, es de 49 años. El 55,6\% de los agricultores de la zona moderada se encuentra en el rango de edad de 51-65 años y en la zona cálida en el rango de 36-50 años.

La participación de mujeres en ambas zonas agroecológicas es más baja que la de los hombres.
Un tercio de los agricultores de la zona moderada y la mitad de la zona cálida no tiene estudios.

Una proporción de $63,89 \%$ se incorpora a la actividad por tradición familiar, más de la mitad trabaja entre 5 y 6 días por semana, y el $100 \%$ no trabaja los domingos.

Las semillas y algunos implementos manuales son los únicos recursos con que cuentan los pequeños agricultores, existiendo precaridad en el tipo de agricultura que practican. La actividad agrícola y pecuaria son las dos principales actividades realizadas en el predio. El 38,89\% de los agricultores considera que la actividad pecuaria es la que más aporta al hogar.

La mitad de los pequeños agricultores mantiene un nivel de venta entre $51 \%$ y $75 \%$ de los productos generados en el predio y solo el 5,6\% mantiene un nivel de ventas superior al $75 \%$.

El 80,56\% de los agricultores son propietarios de sus tierras, cuyo promedio de superficie es 0,83 ha en la zona moderada y de 0,65 ha en la zona cálida. El 44,44\% recurre a donaciones y préstamos de tipo informal para financiar las actividades del predio y solo el $16,67 \%$ utiliza capital propio.

\section{Literatura Citada}

Baumeister, E.

2001. "Peasant initiatives in land reform in Central America", En: Krishna B. Ghimire, editor, Land Reform and Peasant. Livelihoods: The Social Dynamics of Rural Poverty and Agrarian Reform in Developing Countries, ITDG Publishing Londres. pp. 10-195

Cascos, I.

2004. Estadística descriptiva. Disponible en http:// autonomiame diaticaucem.wikispaces.com/file/view/ Estad\%C3\%ADstica+descriptiva.pdf Consultado 15/03/2013.
Dirección de las Estadísticas Demográficas y Sociales (DSDS); Instituto Haitiano de Estadística e Informática (IHSI).

2012. Population totale, population de 18 ans. et plus, Ménages et densités estimés en 2009. Haïti. Disponible en http://www. ihsi.ht/pdf/projection/DOC_POPTLE18_MENEST2012. pdf Consultado 25/12/2013.

Dure, W.

2008. Contribution du secteur primaire a l'économie nationale de 1095 à 2004. Disponible en http://www. memoireonline.com/10/09/2738/mDynamiquedes 
Associations-paysannes-en-Haiumlti-et-le-Developpementlocal-cas-de-1Arrond5. Html Consultado 03/11/2013.

Fanfan, J.

2012. Las asociaciones campesinas y el desarrollo rural en Haití: el caso del movimiento campesino de Papaye. pp. 4-53.

Gédéon, E.

2010. Rapport du diagnostic agraire réalisé dans la section communale de Cap-Rouge dans le cadre du service civique. Document disponible au bureau de la Coordination Régionale des Organisation du Sud Est (CROSE). Jacmel. pp. 18-45 Institut Haitien de Statistique et d'Informatique (IHSI)

2003. $4^{\text {ème }}$ Recensement Général de la Population et de l'Habitat, Port-au-Prince, Haïti. pp. 15-76.

Institut Haïtien de Statistique et d'Informatique (IHSI)

2007. Projections de population totale, urbaine, rurale et économiquement active. Port-au-Prince, Haiti. pp. 3-27.

Institut Haïtien de Statistique et d'Informatique (IHSI)

2009. Population totale, population de 18 ans et plus, Ménages et densités estimés en 2009. Haïti. Disponible en http://www.ihsi.ht/pdf/projection/POPTOTAL\&MENAG DENSESTIM2009.pdf Consultado 12/02/2013.

Instituto Interamericano de Cooperación para la Agricultura (IICA); Comisión Económica para América Latina y el Caribe (CEPAL); Organización de las Naciones Unidas para la Agricultura y la Alimentación

2009. Perspectivas de la agricultura y del desarrollo rural en las Américas 2009. San José, Costa Rica, IICA. Disponible en http://www.eclac.org/publica ciones/xml/8/37598/ ISPAespa\%C3\%Blol_web.pdf Consultado 02/04/2013.

Inter Entreprise S.A. (INESA)

2001. Le café en Haïti : situation actuelle et plaidoyer pour une amélioration de la situation socio-économique des producteurs (document interne). [En Línea]. Disponible en: «http://www.maketradefair.com/fr/assets/francais/ cafeenhaiti.pdf». Consultado 15/12/2013

Jean-Baptiste

2007. Diagnostic technico-économique de la production laitière à Savane Zombi après la mise en place de la Laiterie de la Forêt-des-Pins (document interne), Thiotte/Haïti, Direction régionale du Ministère de l'Agriculture des Ressources Naturelles et du Développement Rural, Port-au-Prince, Haïti. pp. 17-51.

Jean Baptiste, A.

2012. Dynamique des Associations paysannes en Haïti et le Développement local, cas de l'Arrondissement de Belle-Anse.
Port-au-Prince, disponible en : http://www.memoireonline. com/10/09/2738/mDynamiquedes Associationspaysannesen-Haiumlti-et-le-Developpement-local-cas-de-1Arrond5. Html Consultado 10/11/2013.

Ministère de l'Agriculture, des Ressources Naturelles et du Développement Rural (MARNDR)

2010. Plan d'investissement agricole. Document Principal. pp. 6-15.

Ministère de l'Agriculture des Ressources Naturelles et du Développement Rural (MARNDR)

2012. Recensement général de l'agriculture. MARNDR/MICT/ IHSI / CNIGS / FAO / Union Européenne. Port-au-Prince. pp. 19-22.

Organización para la Agricultura y la Alimentación (FAO)

2009. La agricultura de pequeña escala es la solución a la crisis alimentaria, Acción frente a la cumbre de la FAO en el año 2009, Publicado por Semillas de Identidad. Campaña, disponible en http://semillasdeidentidad.blogspot. com/2009/11/la-agricultura-de-pequena-escala-es-la.html Consultado 17/04/2013.

Organización para la Agricultura y la Alimentación (FAO)

2012. Cooperativas Campesinas y seguridad alimentaria: un modelo vigente. Disponible en http://www.fao.org/alc/file/ media/pubs/2012/cooperativas_campesinas.pdf Consultado 24/11/2014.

Pierre, A.

2010. Factores limitantes de la actividad forestal en comunidades rurales de Haití: el caso de la comuna de la Vallée de Jacmel. Pp. 14-33.

Sanon, W.

2010. Estructuración y fortalecimiento de un movimiento agroecológico en Haití. Cayes-Jacmel, Haití. Pp. 28-49.

Torres, $\mathrm{M}$.

2005. Cuadernos estancias de investigación. La identidad profesional docente del profesor de Educación básica en México. Disponible en http://www.crefal. edu.mx/ bibliotecadijital/CEDEAL/acervo_digital/coleccióncrefalcuadernoses estancias.htm Consultado 23/04/2013.

Victor, J.A.

2010. Analyse du cadre légal et institutionnel relatif à la gestion durable des terres. Projet $\mathrm{N}^{\circ}$ 00046489. PNUD/ GEF/MDE. Port-au-Prince, Haiti. Pp. 11-43. 